\title{
DECOMPOSITION THEOREMS FOR VECTOR MEASURES
}

JAMES K. BROOKS

1. Introduction. In this note we consider two theorems for vector measures, each of which is a generalization of a well-known theorem for scalar measures. Theorem 1 extends the Yosida-Hewitt theorem [7], [4, p. 163], which states that a bounded real valued finitely additive (f.a.) measure defined on a Boolean algebra can be decomposed uniquely into a countably additive (c.a.) part and a purely finitely additive (p.f.a.) part. The second theorem, due to Rickart [6, Theorem 4.5], is a Lebesgue decomposition theorem for c.a. vector measures with respect to an outer measure. Several authors have given alternate proofs of a restricted form of this theorem (e.g. see $[3$, p. 189] where the outer measure is replaced by a measure and the total variation of the vector measure is assumed to be finite-a condition not generally satisfied). In 3 we give a short and elementary proof of Rickart's theorem, which represents a considerable simplification of the existing proofs of this result.

Let $\boldsymbol{X}$ be a Banach space over the reals $\boldsymbol{R}$ with first and second conjugate spaces $X^{*}$ and $X^{* *}$; we regard $X$ as a subset of $X^{* *} . \Sigma_{0}$ and $\Sigma$ respectively denote a Boolean algebra and a $\sigma$-algebra of subsets of a set $S$. $\|\mu\|$ denotes the semivariation $[3$, p. 51] of $\mu . \mu$ is bounded if $\|\mu\|(S)<\infty$.

2. Theorem 1. Let $\mu: \Sigma_{0} \rightarrow X$ be bounded and finitely additive. Then $\mu$ can be written uniquely in the form $\mu=\mu_{1}+\mu_{2}$, where the $\mu_{i}: \Sigma_{0} \rightarrow X^{* *}$ are finitely additve and for each $f \in X^{*}:(1) \mu_{1}(\cdot) f: \Sigma_{0} \rightarrow R$ is countably additive and (2) $\mu_{2}(\cdot) f: \Sigma_{0} \rightarrow R$ is purely finitely additive.

Proof. For $f \in X^{*}$ the set function $f \mu$ defined by $(f \mu)(E)=f(\mu(E))$, $E \in \Sigma_{0}$ is f.a. Moreover, since $|f \mu(E)| \leqq\|f\|\|\mu(E)\| \leqq\|f\|\|\mu\|(S), f \mu$ is bounded. By the Yosida-Hewitt theorem $f \mu=\mu_{f, 1}+\mu_{f, 2}$, where $\mu_{f, 1}$ is c.a. and $\mu_{f, 2}$ is p.f.a. For $E \in \Sigma_{0}$ define $F_{i, E}: X^{*} \rightarrow R, i=1,2$, as follows: $F_{i, E}(f)=\mu_{f, i}(E), f \in X^{*}$. It follows from the uniqueness of the decomposition of the scalar measures and Theorem 1.17 in [7] that $\mu_{\alpha f, i}$ $=\alpha \mu_{f, i}$ and $\mu_{f+o, i}=\mu_{f, i}+\mu_{g, i}$, for $f, g \in X^{*}, \alpha \in R$; hence $F_{i, E}$ is linear on $X^{*}$. To show that $F_{i, E} \in X^{* *}$, let $f \in X^{*}$ and let $f \mu=(f \mu)^{+-}(f \mu)^{-}$, $|f \mu|=(f \mu)^{+}+(f \mu)^{-}$be the Jordan decomposition of $f \mu$. Again using the uniqueness of the decomposition of $f \mu$, we have $u_{f, i}=(f \mu)_{i}^{+}-(f \mu)_{i}^{-}$ where the subscripts refer to the c.a. and p.f.a. parts. $\left|F_{i, E}(f)\right|$

Received by the editors April 10, 1968. 
$=\left|\mu_{,, i}(E)\right| \leqq(f \mu)_{i}^{+}(E)+(f \mu)_{i}^{-}(E) \leqq(f \mu)^{+}(E)+(f \mu)^{-}(E)=|f \mu|(E)$ $\leqq 2 \sup _{A E E}|f \mu(A)| \leqq 2 \sup _{A \subseteq E}\|f\|\|\mu(A)\| \leqq 2\|f\|\|\mu\|(E)$. Thus $\left\|F_{i, E}\right\|$ $\leqq 2\|\mu\|(E)$ and $F_{i, E} \in X^{* *}$. Define $\mu_{i}(E)=F_{i, E}, E \in \Sigma_{0}$. It is clear from the construction that $\mu_{1}$ and $\mu_{2}$ have the required properties.

Corollary. Assume $X$ is reflexive and $\mu: \Sigma \rightarrow X$ is bounded and finitely additive. Then $\mu$ can be written uniquely in the form $\mu=\mu_{1}+\mu_{2}$, $\mu_{i}: \Sigma \rightarrow X$, where $\left(1^{\prime}\right) \mu_{1}$ is countably additive and $\left(2^{\prime}\right) f \mu_{2}$ is purely finitely additive for each $f \in X^{*}$.

Proof. Let $\mu_{i}$ be the components of $\mu$ given in the above theorem. Since $f \mu_{1}(\cdot)=\mu_{1}(\cdot) f$ is c.a. for $f \in X^{*}, \mu_{1}$ is weakly countably additive in addition to being f.a. By the Pettis theorem [4, p. 318] $\mu_{1}$ is c.a., and the result follows.

REMARK. For a reflexive space $\boldsymbol{X}$, the existence of a large number of bounded f.a. $X$-valued measures on $\Sigma$, hence measures satisfying $\left(2^{\prime}\right)$, can be deduced as follows. Let $Y=M_{X^{*}}(\Sigma)$ be the Banach space of totally measurable $X^{*}$-valued functions (i.e. uniform limits of simple functions) with the uniform norm. Since $Y^{*}$ is isomorphic to the set of all f.a. $X$-measures on $\Sigma$ having finite total variation [2], the Hahn-Banach theorem implies that for each $0 \neq g \in Y$ and $\alpha \in R$, there exists a f.a. $X$-measure $\mu$ such that $\int_{S} g d \mu=\alpha$, where the integral is defined in [3].

3. The Lebesgue decomposition. Definitions. Let $\beta$ be an outer measure defined on $\Sigma$. Then $\mu: \Sigma \rightarrow X$ is $\beta$-continuous if $\beta(E) \rightarrow 0 \mathrm{im}-$ plies $\mu(E) \rightarrow 0 . \mu$ is $\beta$-singular if there exists an $E^{*} \in \Sigma$ such that $\beta\left(E^{*}\right)=0$ and $\mu(E)=\mu\left(E \cap E^{*}\right), E \in \Sigma$.

Theorem 2. Let $\mu: \Sigma \rightarrow X$ be countably additive and let $\beta$ be an outer measure defined on $\Sigma$. Then $\mu$ can be decomposed uniquely into the form $\mu=\mu_{1}+\mu_{2}$, where $\mu_{1}$ and $\mu_{2}$ are countably additive, $\mu_{1}$ is $\beta$-continuous, and $\mu_{2}$ is $\beta$-singular.

Proof. The uniqueness of $\mu_{1}$ and $\mu_{2}$ is obvious. By a theorem due to Bartle, Dunford, and Schwartz [1], there exists a finite nonnegative c.a. measure $\lambda$ defined on $\Sigma$ such that $\mu$ is $\lambda$-continuous (see [5] for an elementary proof of this result). $\lambda$ can be decomposed uniquely into a $\beta$-continuous part $\lambda_{c}$ and a $\beta$-singular part $\lambda_{s}$. This can be seen by examining the proof of the classical Lebesgue decomposition theorem in $[4, p .132]$. The argument used there remains valid if the appropriate measure appearing in the proof is replaced by an outer measure. Consequently, there exists a set $E^{*} \in \Sigma$ such that $\beta\left(E^{*}\right)=0$ and $\lambda_{s}(E)=\lambda\left(E \cap E^{*}\right), \quad \lambda_{c}(E)=\lambda\left(E \cap\left(S-E^{*}\right)\right)$. Let $\mu_{1}(E)$ 
$=\mu\left(E \cap\left(S-E^{*}\right)\right)$ and $\mu_{2}(E)=\mu\left(E \cap E^{*}\right)$. Obviously $\mu_{2}$ is $\beta$-singular. Now if $\beta\left(E_{n}\right) \rightarrow 0$, then $\lambda\left(E_{n} \cap\left(S-E^{*}\right)\right)=\lambda_{c}\left(E_{n}\right) \rightarrow 0$; hence $\mu_{1}\left(E_{n}\right)$ $=\mu\left(E_{n} \cap\left(S-E^{*}\right)\right) \rightarrow 0$, and $\mu_{1}$ is $\beta$-continuous. The conclusion of the theorem now follows.

ADDED IN PROOF. The classical Lebesgue decomposition theorem (even with respect to an outer measure) can be proved in a straightforward fashion that avoids the Radon-Nikodym theorem. This method was used by the author to decompose set functions of a more general type than the ones considered here (cf. An integration theory for set-valued measures. I, Bull. Soc. Roy. Sci. Liège, no5-8 (1968), 312-319). We shall sketch the proof. Let $\lambda$ and $\beta$ be as in the above proof. Define $\mathfrak{N}=\{E \in \Sigma: \beta(E)=0\} ; \eta=\sup \lambda(E), E \in \Re$. By using the method of exhaustion, one can construct a set $E^{*}$ such that $E^{*} \in \mathfrak{N}$ and $\lambda\left(E^{*}\right)=\eta$. It follows that if $E \in \Re$, then $\lambda\left(E-E^{*}\right)=0$. Then define $\lambda_{s}$ and $\lambda_{c}$ to be the restrictions of $\lambda$ to $E^{*}$ and $S-E^{*}$ respectively.

\section{BiBLIOGRAPHY}

1. R. G. Bartle, N. Dunford and J. Schwartz, Weak compactness and vector measures, Canad. J. Math. 7 (1955), 289-305.

2. N. Dinculeanu, Linear operations on spaces of totally measurable functions, Rev. Roumaine Math. Pures Appl. 10 (1965), 1493-1524.

3. - Vector measures, Pergamon Press, New York, 1967.

4. N. Dunford and J. Schwartz, Linear operators, Part I: General theory, Interscience, New York, 1958.

5. G. Gould, Integration over vector-valued measures, Proc. London Math. Soc. 15 (1965), 193-225.

6. C. E. Rickart, Decomposition of additive set functions, Duke Math. J. 10 (1943), 653-665.

7. K. Yosida and E. Hewitt, Finitely additive measures, Trans. Amer. Math. Soc. 72 (1952), 46-66.

UNIVERSITY OF FLORIDA 